\title{
Rheological Study of Polymers Used as Viscosity Index Improvers for Automotive Lubricant Oils
}

\author{
Ana Paula Pena Almeida, Ana Paula Lelis Rodrigues de Oliveira, \\ Cynthia D'Ávila Carvalho Erbetta, Ricardo Geraldo de Sousa, \\ Roberto Fernando de Souza Freitas, Maria Elisa Scarpelli Ribeiro e Silva* \\ Polymer Science and Technology Laboratory, Chemical Engineering Department, Engineering School, Federal \\ University of Minas Gerais, Belo Horizonte, Brazil \\ Email: ${ }^{*}$ elisa@deq.ufmg.br
}

Received 23 May 2014; revised 21 June 2014; accepted 12 July 2014

Copyright (C) 2014 by authors and Scientific Research Publishing Inc.

This work is licensed under the Creative Commons Attribution International License (CC BY). http://creativecommons.org/licenses/by/4.0/

(c) (i) Open Access

\begin{abstract}
In this work, a rheological study of automotive lubricant oils, neutral light paraffinic (NLP) and neutral medium paraffinic (NMP), additivated with two commercial viscosity index improversVIIs, based on hydrogenated poly (isoprene-co-styrene), at different concentrations, was carried out. The study included the determination of the kinematic viscosity of the mixtures, the calculation of the viscosity indexes and their behavior when subjected to various rheometric experiments, including stress sweep, steady state flow, creep and recovery, and temperature ramp oscillatory tests. The obtained results showed the efficacy of the additives as viscosity index improvers, enabling a significant increase in the viscosity index of the pure oils at relatively low concentrations. The determination of the storage ( $G^{\prime}$ ) and of the loss ( $\left.G^{\prime \prime}\right)$ moduli for the mixtures oil/VII additives showed a large predominance of the viscous effect over the elastic one. Except when under low shear stress, the mixtures showed a pseudoplastic behavior, with the flow curves being adjusted to fit the Ostwald-de-Waele model (Power Law), with negative flow behavior indexes.
\end{abstract}

\section{Keywords}

Viscosity Index Improvers, Automotive Lubricants, Rheology, Viscosity Index, Polymer Additives

\section{Introduction}

The demand for technological innovations in the lubricant oil sector has steadily increased with the evolution of

*Corresponding author.

How to cite this paper: Almeida, A.P.P., Oliveira, A.P.L.R., Erbetta, C.D.C., Sousa, R.G., Freitas, R.F.S. and Silva, M.E.S.R. (2014) Rheological Study of Polymers Used as Viscosity Index Improvers for Automotive Lubricant Oils. Journal of Modern Physics, 5, 1085-1093. http://dx.doi.org/10.4236/jmp.2014.512110 
automotive engines. Currently, many lubricant properties have been reinforced or created by means of chemical polymeric additives, in the constant search for improved performance, efficiency, durability, and reduction of emissions and operational costs of automotive engines.

Brazil has had a distinguished role in this area for having cutting-edge technology in oil exploration and refining as well as for being a promising market for the sale of lubricant oils. In 2009, the country consumed 3.2\% of the total volume of the product sold worldwide and, according to recent research, the national demand is expected to increase significantly until 2019 [1]. Considering this scenario, the country has the opportunity to develop its knowledge about the technology of polymeric chemical additives for automotive lubricant oils.

Viscosity index (VI) represents the effect of temperature on the kinematic viscosity of lubricant oils, and was initially proposed by Dean and Davis in 1929 [2]. It measures the stability of the kinematic viscosity with temperature. The higher the VI, the more stable the kinematic viscosity with temperature. For petroleum lubricant oils, a VI slightly above 100 is almost the maximum limit attainable by economical refining processes [3]. Any additional increase in the VI can only be achieved with the addition of polymers known as Viscosity Index Improvers (VIIs).

Viscosity index improvers (VIIs) are among the most important additives for lubricant oil formulation [4]. Polymers usually used as VIIs are olefins copolymers (OCPs), poly (alkyl methacrylates) (PAMAs), hydrogenated copolymers of (styrene-co-butadiene) (HSD) and their modifications, hydrogenated copolymers of (styreneco-isoprene) (HSI) and their modifications, along with the star-shaped polymers with a radial hydrogenated poly (isoprene) base (HRI) [5]-[7].

This study was motivated by the need for a greater understanding of the characteristics of the polymers used as viscosity index improvers in paraffinic oil. This paper reports the rheological study of automotive lubricant oils, neutral light paraffinic (NLP) and neutral medium paraffinic (NMP), additivated with two commercial VIIs, at different concentrations. The study included the determination of the kinematic viscosity of the mixtures, the calculation of the viscosity indexes and their behavior when subjected to various rheometric tests.

\section{Experimental}

The experiments carried out in this work involved the preparation of the mixtures (oil plus VII), determination of the kinematic viscosities, calculation of the viscosity indexes, and rheometric tests.

\subsection{Materials}

Samples of neutral light and neutral medium paraffinic oils and samples of oils with two VII additives based on hydrogenated poly (isoprene-co-styrene) were used for this study. The commercial VIIs used were codified as A2 and A5.

\subsection{Preparation of Samples}

The procedure used for the preparation of the samples was dissolution of the VIIs in neutral light and in neutral medium paraffinic oils, with manual stirring without heating, utilizing a glass rod for the initial homogenization of the mixture. Subsequently, mechanical stirring was used (IKA-MOD. RW 20n shaker) at $960 \mathrm{rpm}$ for 20 minutes, increasing, thereafter, to $2400 \mathrm{rpm}$ for 60 minutes, without heating. The mixtures were prepared at three mass concentrations of each VII additive- $1 \%, 2 \%$ and $4 \%$. The kinematic viscosities of the mixtures with $1 \%$ and $2 \%$ concentration of additive were determined, and their viscosities indexes were, then, calculated. For the rheometric tests, the mixtures with $2 \%$ and $4 \%$ concentration of additive were used.

\subsection{Determination of Kinematic Viscosity and Viscosity Index}

The kinematic viscosities of the paraffinic oils and of the mixtures oils/VII additives were determined, according to ASTM D-445 and ASTM D- 446 , at $40^{\circ} \mathrm{C}$ and at $100^{\circ} \mathrm{C}$, using a Herzog viscometer. From the obtained kinematic viscosities, the viscosity indexes were calculated, according to ASTM D-2270.

\subsection{Rheometric Tests}

The behavior of the paraffinic oils and of the mixtures oils/VII additives when subjected to rheometric tests was 
studied using a rheometer model AR-G2 Rheometer (TA Instruments), concentrical cylinders geometry. Stress sweep test, in the range of 0.001 to $1063 \mathrm{~Pa}$ at $25^{\circ} \mathrm{C}$ and at a $1 \mathrm{~Hz}$ frequency, was used for the determination of the linear viscoelasticity region. From steady state flow tests, at $25^{\circ} \mathrm{C}$, stress-strain curves were obtained and the viscosity was determined. In this experiment, the strain rate varied between $5.316 \times 10^{-3}$ and $600 \mathrm{~Pa}$.

For the evaluation of the viscoelastic behavior of the samples, creep and recovery tests were carried out in two steps: 1 ) the material was subjected to a constant shear stresses $(\tau)$ of 1 and of $10 \mathrm{~Pa}$, for a period of 15 minutes $\left(t_{1}\right)$; 2$)$ the tension was, then, instantly removed and, for a period of 10 minutes $\left(t_{2}\right)$, the recovery of the sample was observed. The values for the shear stress (within the Newtonian plateau) were selected from the steady state flow experiments. Throughout the test, the strain $(\gamma)$, as a function of time, was measured.

Temperature ramp oscillatory tests were conducted, in order to evaluate the viscoelastic behavior of the samples, with continuously increasing temperature. The temperature range of the experiments varied from $25^{\circ} \mathrm{C}$ to $150^{\circ} \mathrm{C}$, at a heating rate of $5^{\circ} \mathrm{C} \cdot \mathrm{min}^{-1}$, maintaining the tension and the frequency constants at $1 \mathrm{~Pa}$ and $1 \mathrm{~Hz}$, respectively. The storage or elastic (G') and the loss or viscous (G”) moduli, as well as the complex viscosity $\left(\eta^{*}\right)$, were determined, as a function of temperature.

\section{Results and Discussion}

The kinematic viscosities and the viscosity indexes of the pure oils and of the mixtures with the two addditives (A2 and A5), at $1 \%$ and 2\% mass concentration, were determined and the results are presented and discussed in this section. Also, the results obtained from the rheometric tests, for the mixtures with $2 \%$ and $4 \%$ mass concentration of additives, are shown and analyzed considering the effect of the polymer addition to the oils, taking into account their structure, their concentration and other important parameters which will determine their rheological behavior.

\subsection{Determination of Kinematic Viscosity and Viscosity Index}

As seen, from the results presented in Table 1, the kinematic viscosities of MNP oils, at both temperatures, are superior to those of LNP oils, which is in accordance with the specifications for these products [8]. At both temperatures, the kinematic viscosities of the mixtures (oil + VII) are higher than those of pure oils, and they increase with VII concentration, this dependence being more dramatic as the VII concentration increases. Also, it can be noted that, the higher the temperature, the lower the kinematic viscosity.

It is known that the viscosity of a polymeric solution depends on the size of polymer chains, on the extent of their intra and intermolecular interactions, on the extension of polymer and solvent molecules interactions, on polymer concentration and on the nature of the solvent [3]. From the results obtained in the present work, it can be observed that both oils (LNP and MNP) are good solvents for both VII additives (A2 and A5), due to the facility of VIIs dissolution in the oils and to the stability of the mixtures, observed over time. In a good solvent, the polymeric chains are in an uncoiled state. An increase in temperature will cause a weakening in the polymersolvent bonds, enabling an increase of the intra and intermolecular interactions (polymer-polymer interactions), resulting in the coiling of the polymeric molecules, resulting in a smaller hydrodynamic volume, with the con-

Table 1. Kinematic viscosities, at, $40^{\circ} \mathrm{C}$ and at $100^{\circ} \mathrm{C}$ of pure LNP and MNP oils and of their mixtures with VIIs A2 and $\mathrm{A} 5$, at $1 \%$ and $2 \%$ mass concentration.

\begin{tabular}{|c|c|c|c|c|c|}
\hline \multirow{2}{*}{ Temperature, ${ }^{\circ} \mathrm{C}$} & \multicolumn{5}{|c|}{ Pure oil and mixtures oil + VII } \\
\hline & [VII], \% & $\mathrm{LNP}+\mathrm{A} 2, \mathrm{~mm}^{2} \cdot \mathrm{s}^{-1}$ & $\mathrm{LNP}+\mathrm{A} 5, \mathrm{~mm}^{2} \cdot \mathrm{s}^{-1}$ & $\mathrm{MNP}+\mathrm{A} 2, \mathrm{~mm}^{2} \cdot \mathrm{s}^{-1}$ & $\mathrm{MNP}+\mathrm{A} 5, \mathrm{~mm}^{2} \cdot \mathrm{s}^{-1}$ \\
\hline \multirow{3}{*}{40} & 0 & 30.27 & 30.27 & 52.58 & 52.58 \\
\hline & 1.0 & 79.75 & 91.66 & 125.80 & 132.10 \\
\hline & 2.0 & 203.90 & 257.20 & 341.60 & 434.90 \\
\hline \multirow{3}{*}{100} & 0 & 5.21 & 5.21 & 7.30 & 7.30 \\
\hline & 1.0 & 12.45 & 14.20 & 15.92 & 16.73 \\
\hline & 2.0 & 28.65 & 35.47 & 38.85 & 48.51 \\
\hline
\end{tabular}


sequent reduction in viscosity.

It is worthy noting, also from the results presented in Table 1, that the thickening effect of A5 VII was greater than that of A2 VII. It is known that, in hydrogenated styrene-isoprene copolymers, the macromolecules are bonded by styrene blocks which, in a solution, are not well dissolved by the oil, forming a type of "loose network" [9]. The effect of network formation implies in the thickening of the oil, this effect being proportional to the concentration of styrene blocks. Thus, the observed difference in the thickening effect of the VIIs used in this work, can be associated with possible differences in the molecular structure of the polymers, with respect to the amount of styrene blocks.

Based on the data of kinematic viscosities, at $40^{\circ} \mathrm{C}$ and $100^{\circ} \mathrm{C}$, the viscosity indexes were calculated, and the results are shown in Table 2. The viscosity indexes obtained for the LNP and for the MNP oils, 102 and 98, respectively, are in accordance with the values expected for lubricant oils (paraffinic petroleum), $\mathrm{C}_{24}$ to $\mathrm{C}_{50}$, deparaffinated, dearomatized by solvent and hydrogenated [8] [10]. It can be seen, from the data presented in Table 2, that the viscosity indexes increase with the addition of VII and, also, the greater the VII concentration, the greater the VI. Also, this effect is more significant when the polymers are added to the LNP oil and, in addition, this effect is more pronounced when the A5 VII is added compared to the A2 VII.

According to [11], when the polymer is added to the paraffinic oil in small concentrations, usually around 0.5 to $2 \%$ in mass percentage, it hardly affects the temperature dependence of viscosity of the paraffinic oil, except when there is a strong interaction between the VII and the oil or if a phase transition occurs. For the systems studied in the present work, the results shown in Table 1, and Table 2, as the addition of VIIs contributed to the increasing of the viscosity of the paraffinic oil, both at low and high temperatures, as well as a VI increase, and no phase transition was observed, suggest that there is a strong interaction between the VIIs and the oils, implying in a bigger hydrodynamic volume.

According to [12], lubricating oils containing OCPs and PAMAs copolymers as additives show a lesser variation of viscosity with increasing temperatures when compared to pure paraffinic oil. The VIs of oils containing additives were larger than 140 , reaching a maximum value of 154 when $2 \%$ of PAMA additive was added. Jukić and co-authors [13] studied the effect of the increased viscosity index of paraffinic oil with added styrene/dodecil/octadecyl metacrilate terpolymers, and observed that all terpolymer/paraffinic mineral oil systems, with 5\% mass concentration, showed a VI greater than 150 .

In the present study, significant increases of the VIs were observed, reaching high values for mixtures at relatively small concentrations, $1 \%$ and $2 \%$ in mass. The fact that the A5 VII is more effective in increasing the VI compared to the A2 VII can be explained based on the formation of a "loose network", due to the styrene blocks, earlier discussed, and allows one to use a smaller quantity of this additive for an specific selected VI.

\subsection{Analysis of Rheological Behavior from the Rheometric Tests}

\subsubsection{Stress Sweep Tests}

The linear viscoelasticity region was determined through stress sweep tests for the evaluation of the resistance of the material to deformation until the collapse of its structure. It is necessary that the stress applied in this region induces a proportional deformation response [14]. All mixtures oils (LNP and MNP)/VIIs additives (A2 and A5), at different concentrations, presented a significant region of linear viscoelasticity, which means that the systems present a good stability to deformation. Through this test, it was also possible to observe, as expected, a predominance of the viscous behavior over the elastic one, since the quantity of oil present in the samples are much greater than the quantity of polymer added.

Table 2. Viscosity index (VI) of the pure LNP and MNP oils and of their mixtures with VIIs A2 and A5, at $1 \%$ and $2 \%$ mass concentration.

\begin{tabular}{ccccc}
\hline & \multicolumn{4}{c}{ Pure oil and mixtures oil + VII } \\
\cline { 2 - 5 } [VII], \% & LNP + A2 & LNP + A5 & MNP + A2 & MNP + A5 \\
\hline 0 & 102 & 102 & 98 & 98 \\
1.0 & 154 & 160 & 134 & 137 \\
2.0 & 180 & 187 & 164 & 172 \\
\hline
\end{tabular}




\subsubsection{Steady State Flow Tests}

From the steady state flow tests, stress-strain curves were obtained, as shown in Figure 1, and it was possible to obtain the respective viscosity curves, as shown in Figure 2. It was observed that the samples present a nonNewtonian behavior and, with the aid of the rheometer software, the obtained flow curves presented a best fit to Ostwald-de-Waele model (Power Law). The Power Law flow behavior indexes obtained were smaller than 1, in accordance with those expected for pseudoplastic fluids. Nevertheless, it can be observed that, at low shear stress values, the viscosity of the samples remains constant, and that it decreases as the shear stress values increase. This behavior can be explained considering that, in a low shear stress region, the polymeric molecules remain randomly dispersed, due to the Brownian movement, despite the shearing orientation initial effects. So, at very low shear stress, the pseudoplastic fluids behave as Newtonian fluids, having a viscosity $\left(\eta_{0}\right)$ independent of the shear stress. When the shear stress $(\gamma)$ increases to a point in which shearing, which induces the polymeric molecule orientation, exceeds the randomizing effect of the Brownian movement, the viscosity decreases, that is, a pseudoplastic behavior.

\subsubsection{Creep and Recovery Tests}

By analyzing the results obtained from the creep and recovery tests, it can be observed that the compliance (strain/stress ratio) is independent of the applied stress, as expected for the linear viscoelasticity region, as seen in Figure 3 and Figure 4. Additionally, it is possible to observe that an increase in additive concentration (both for A2 and A5), for the whole time interval of the tests, results in a decrease in the compliance value. This behavior can be explained by the presence of the polymer which, even in low concentrations, offers greater resistance to deformation for an applied stress. The difference in behavior between the systems becomes even more evident when the compliance values of the oils with additives are compared with the values of the pure oils.

\subsubsection{Temperature Ramp Oscillatory Tests}

In Figure 5, it is shown a typical curve of the storage or elastic (G') and of the loss or viscous (G”) moduli and

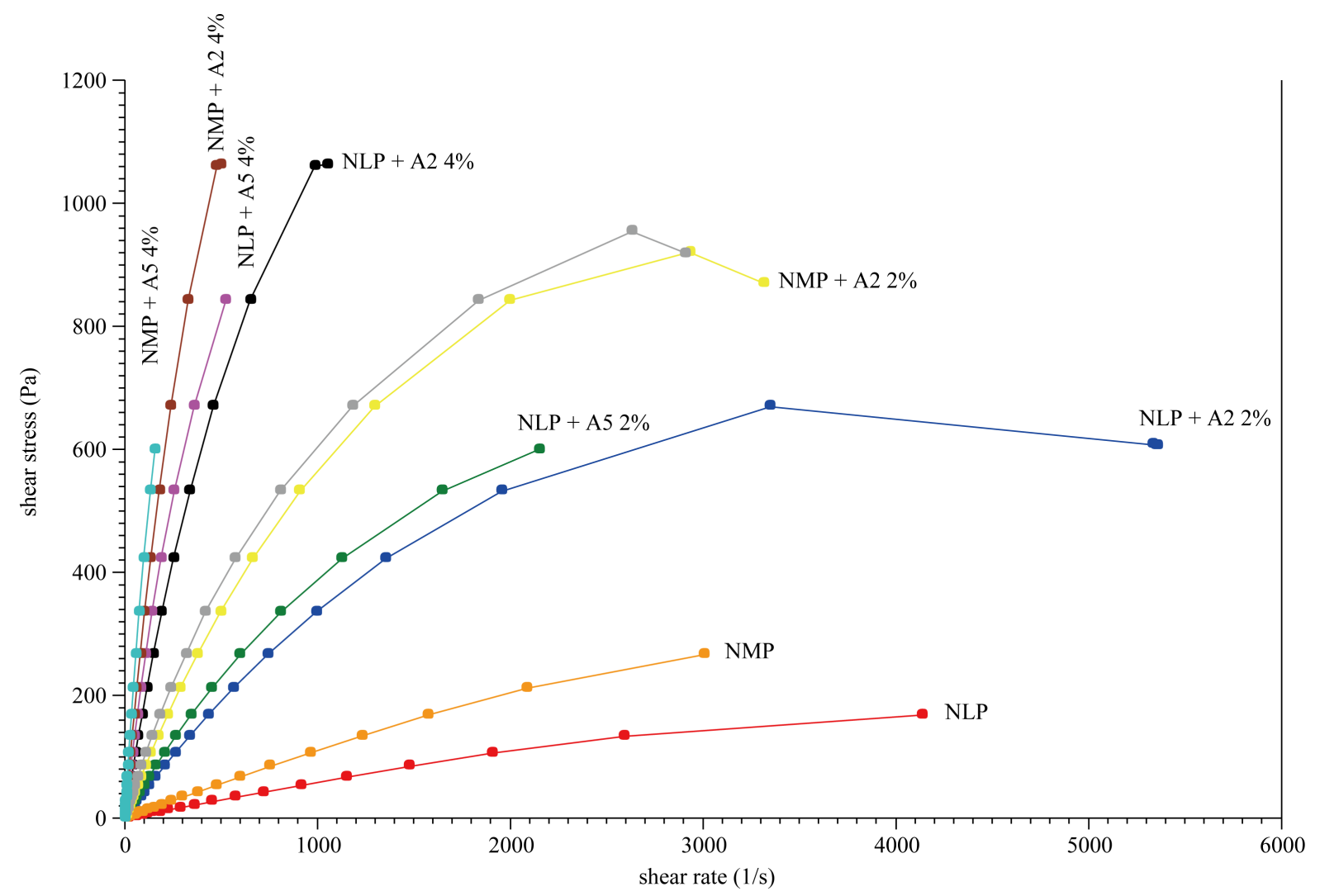

Figure 1. Stress-strain curves obtained from steady state flow tests. 


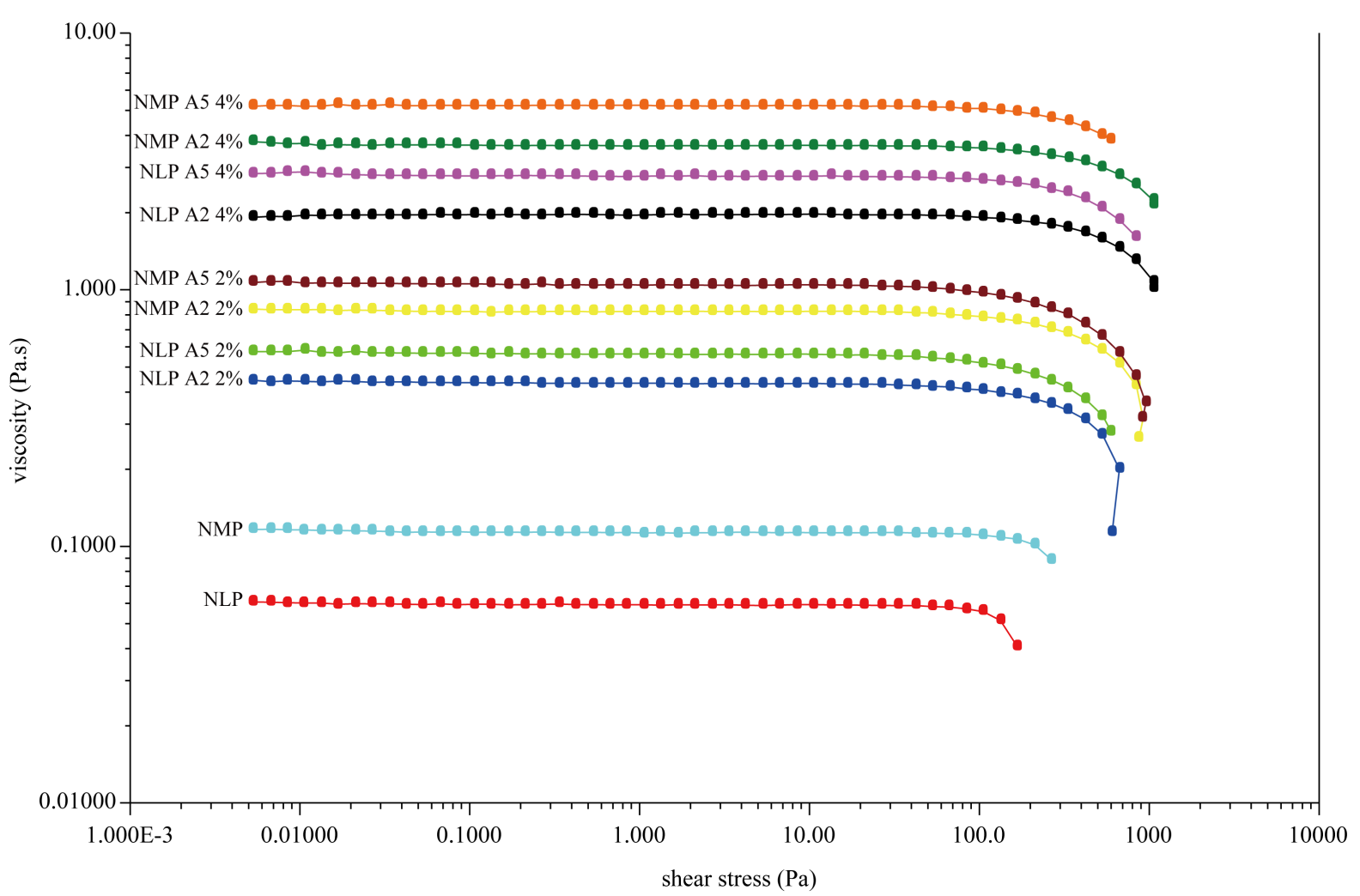

Figure 2. Viscosity as a function of shear stress.

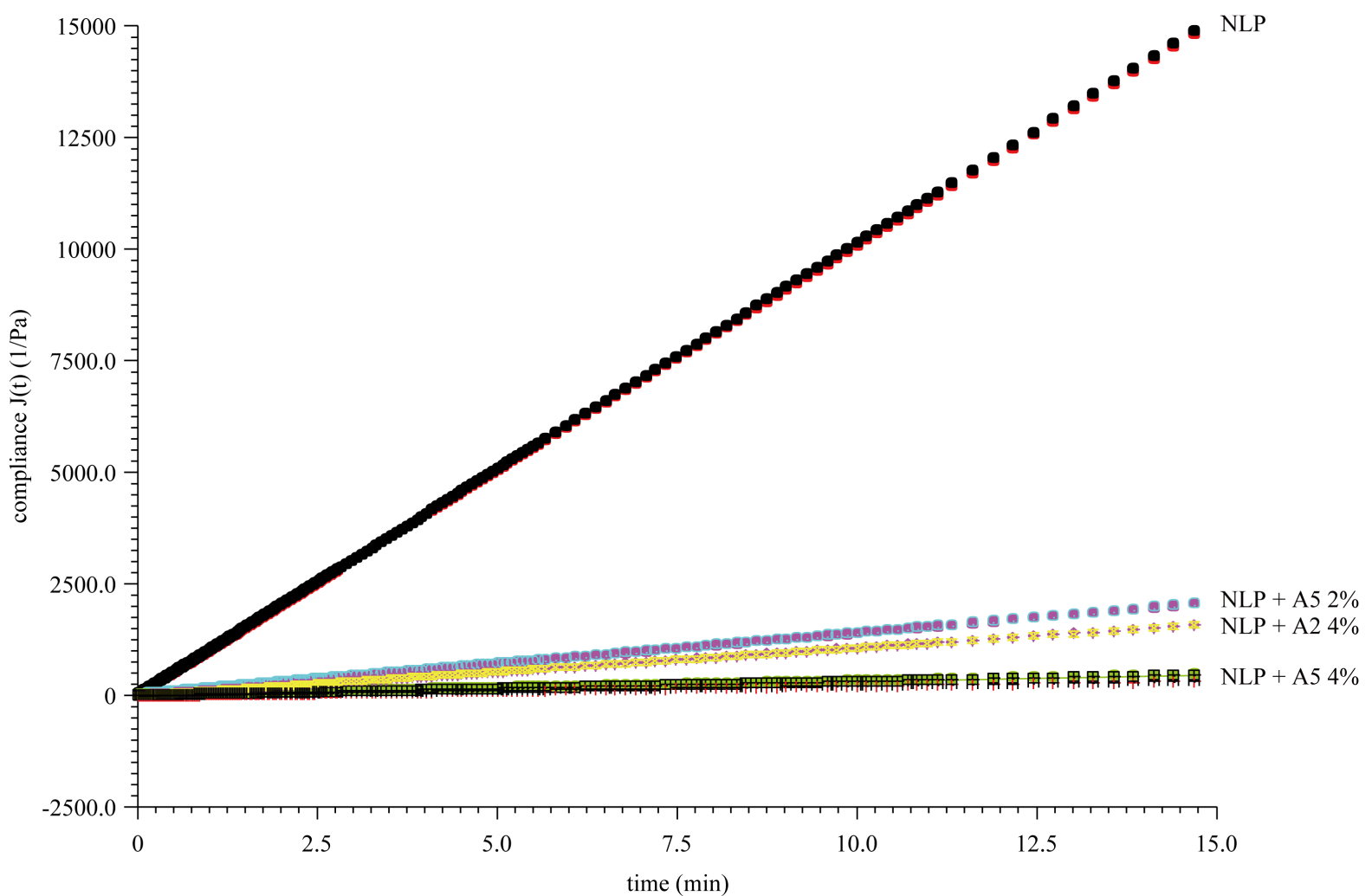

Figure 3. Compliance versus time curves for samples of pure LNP and with additives (shear stress of 1 and of $10 \mathrm{~Pa}$ ). 


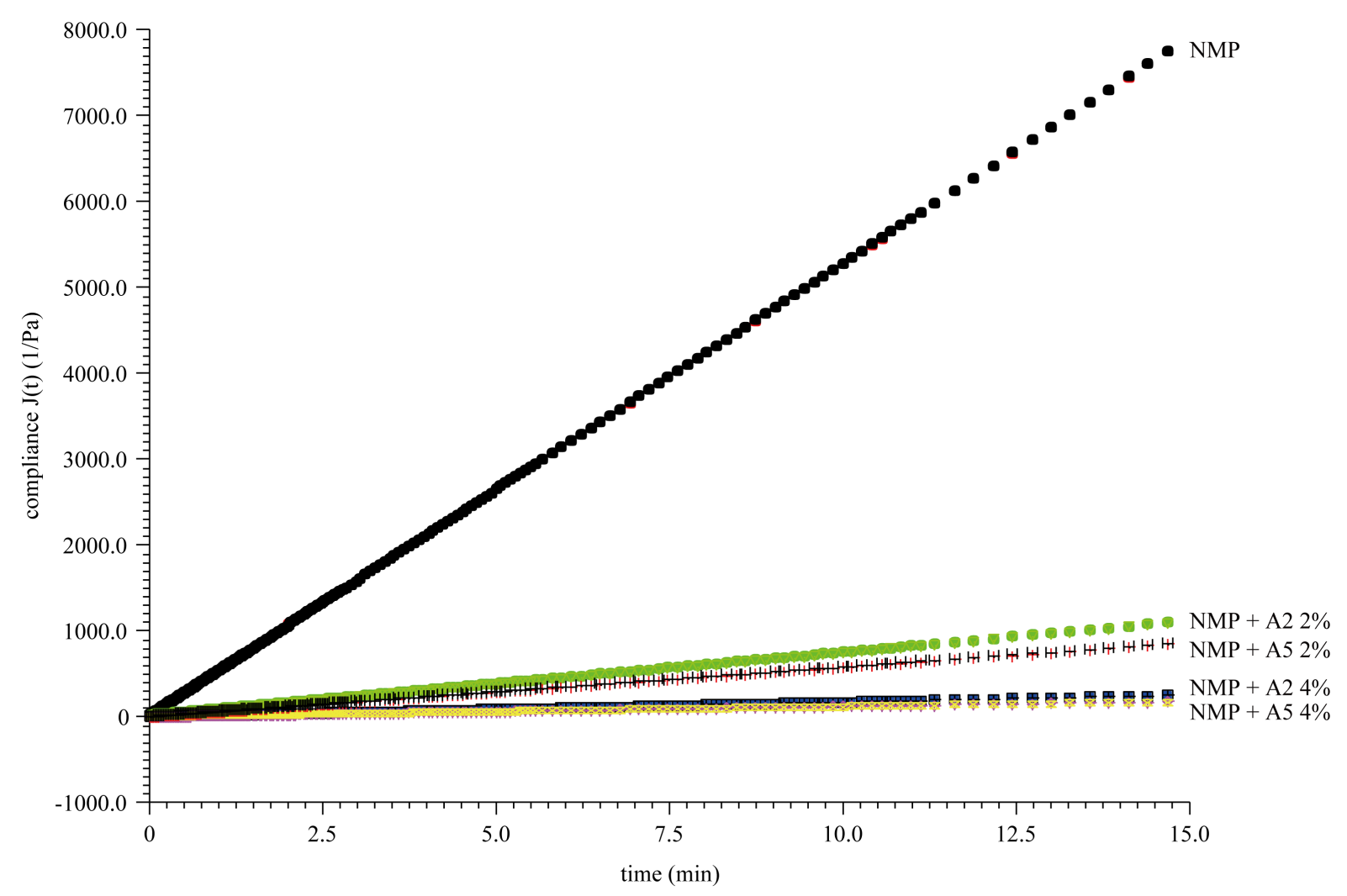

Figure 4. Compliance versus time curves for samples of pure MNP and with additives (shear stress of 1 and of $10 \mathrm{~Pa}$ ).

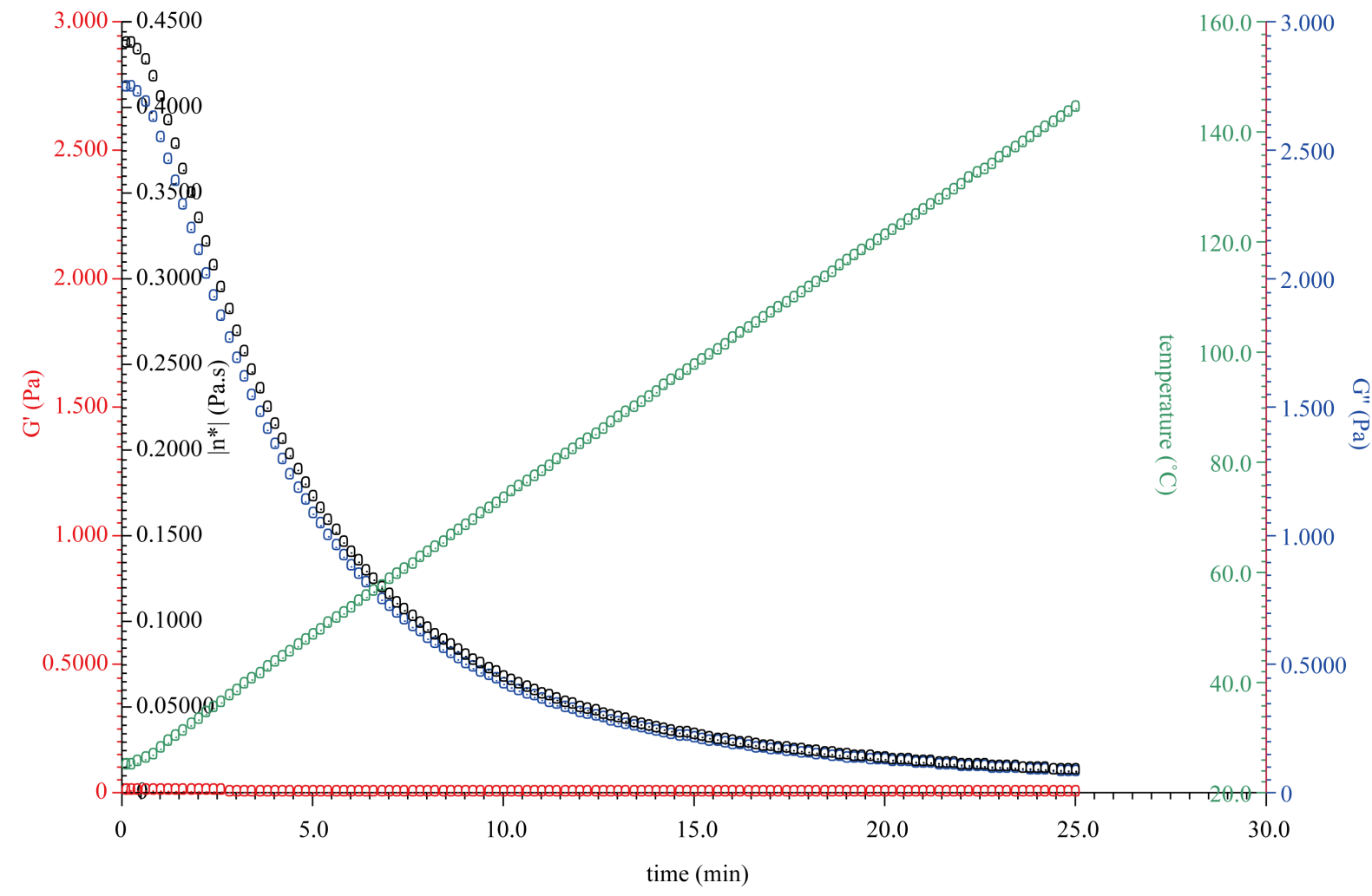

Figure 5. (G'), (G”) and ( $\left.\eta^{*}\right)$ curves for LNP + A2 VII (2\%) as a time function. 
of the complex viscosity $\left(\eta^{*}\right)$ as a function of time, obtained through the temperature ramp oscillatory test. In this figure, it is possible to observe that the visocus effect largely prevails over the elastic one, a result which corroborates those obtained from the stress sweep tests.

In Figure 6 and Figure 7 it is shown the dependency of the complex viscosity on temperature for the LNP and LNP + VII additives samples studied. For the MNP and MNP + VII additives, a similar behavior was observed. An increase in temperature induced a reduction of $\eta^{*}$ of the samples of light neutral, and medium neutral paraffinic mineral oils containing additives, a more pronounced effect in samples with higher additive concentration. By zooming the curves for the $100^{\circ} \mathrm{C}$ to $150^{\circ} \mathrm{C}$ range, as seen in Figure 7, it is shown that the complex viscosities of the mixtures, for all polymer concentrations, are higher than those of the pure oils, which implies in the efficacy of the A series additives as viscosity index improvers.

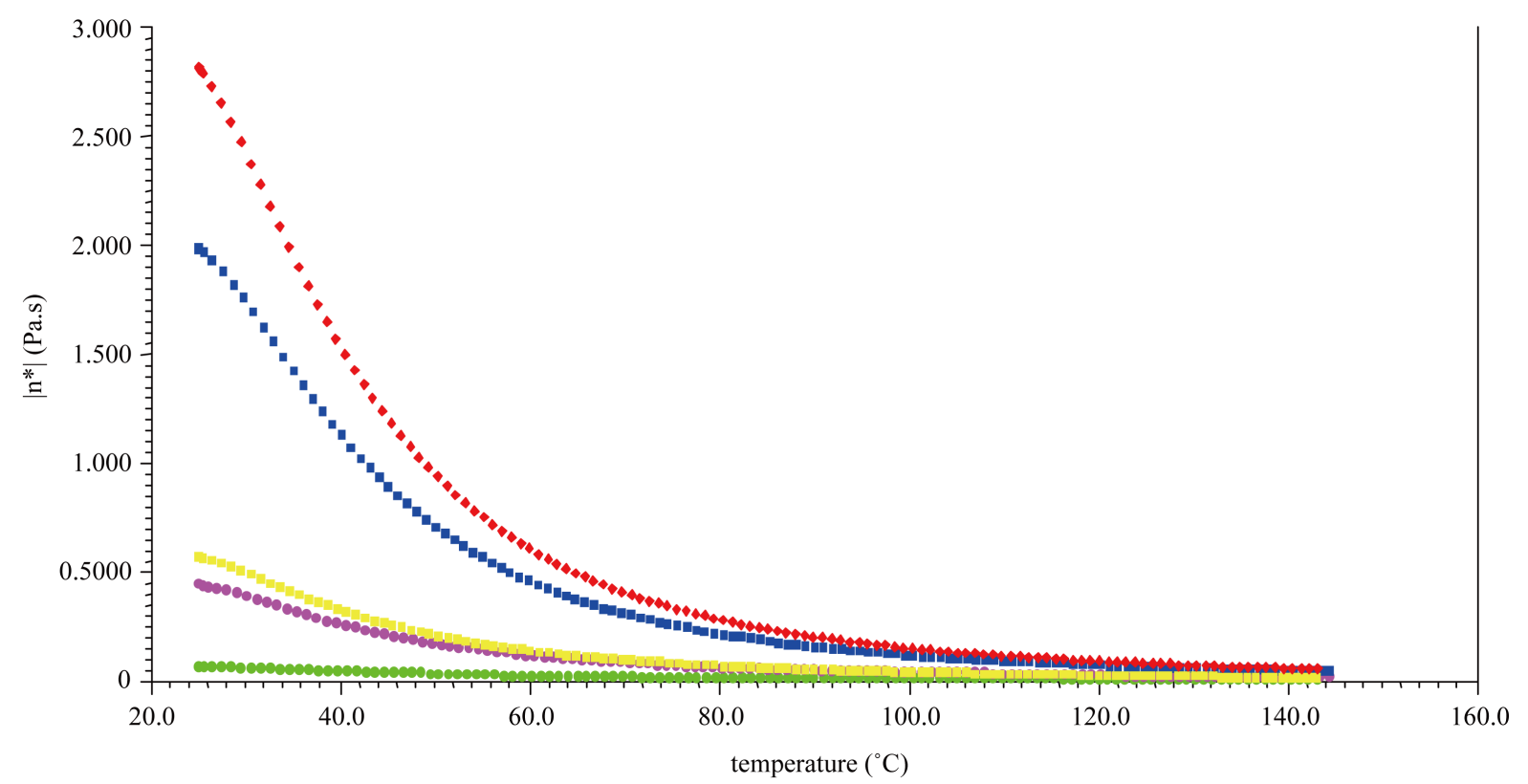

Figure 6. $\left(\eta^{*}\right)$ curves as a function of temperature for LNP samples (with and without additives).

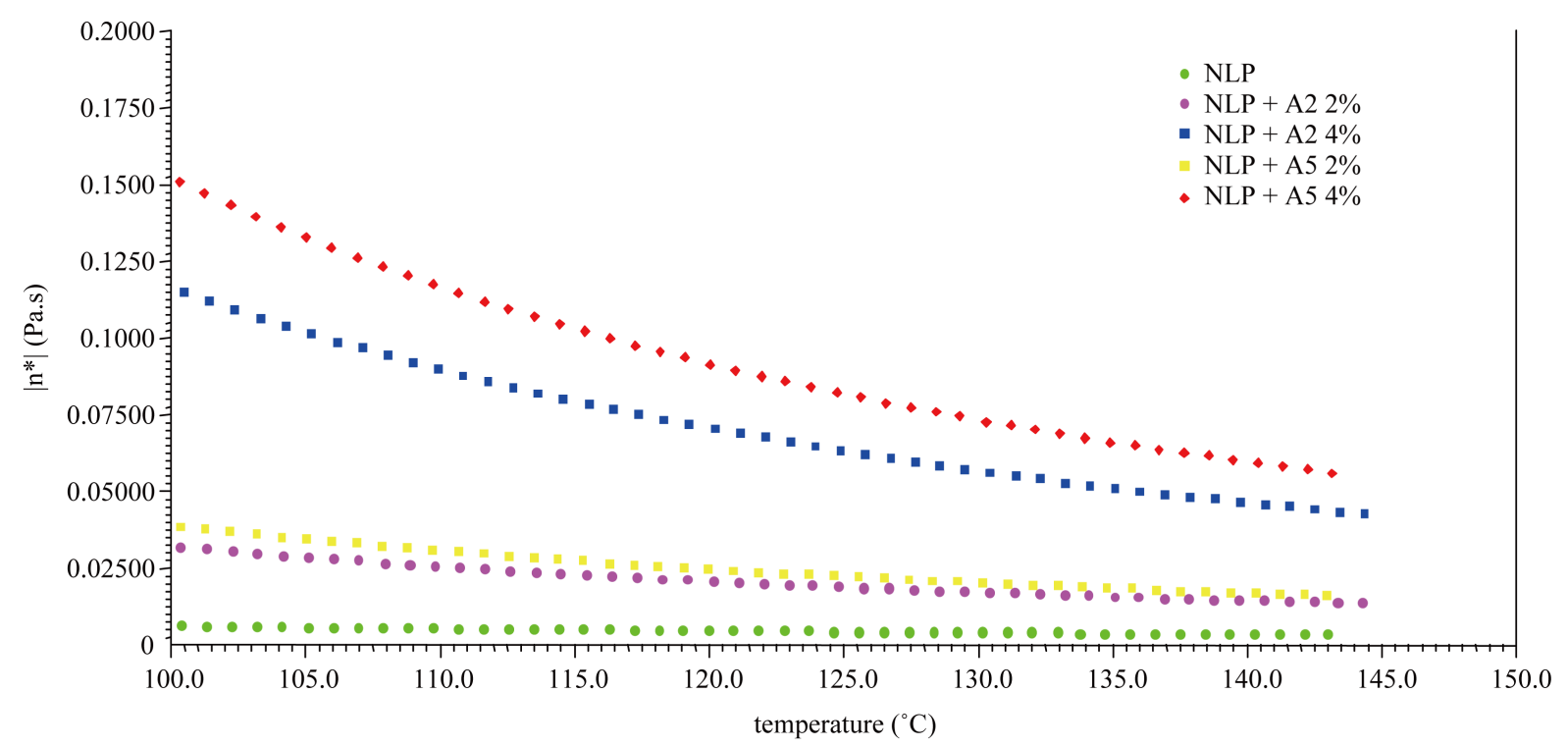

Figure 7. $\left(\eta^{*}\right)$ curves as a function of temperature for LNP samples (with and without additives) for the $100^{\circ} \mathrm{C}$ to $150^{\circ} \mathrm{C}$ range. 


\section{Conclusion}

Both A series (A2 and A5) of additives, based on hydrogenated poly (isoprene-co-styrene), used in this work, are effective as viscosity index improvers, as shown by the determination of the viscosity indexes and by different rheometric tests. Light neutral paraffinic and medium neutral paraffinic oils are good solvents for the VIIs studied. The additives were more effective when dissolved in LNP oil, resulting in a greater percentage increase in the viscosity index, as compared to the dissolution in MNP oil. The additives showed different thickening effect, the A5 presenting a greater effect. This result was interpreted considering the formation of a loose network, due to the styrene blocks, which present low solutbility in paraffinic oil. The mixtures oil/VIIs additives showed a pseudoplastic behavior, with the flow curves being adjusted to fit the Ostwald-de-Waele model (Power Law), with negative flow behavior indexes. However, for low shear stresses, the mixtures showed a Newtonian behavior. It was observed that the VIIs decrease the compliance and, this effect is a function of VII additive concentration. This result was attributed to the resistance of the polymer to deformation, for an applied stress. It was observed, by different rheometric tests (temperature ramp oscillatory and stress sweep), that the visocus effect largely prevails over the elastic one, for mixtures oil/VIIs additives.

\section{Acknowledgements}

The authors thank the Polymer Science and Tecnology Laboratory of Federal University of Minas Gerais, Petrobras, CNPq, CAPES and FAPEMIG for all support for this research.

\section{References}

[1] Barros, G. (2010) Mercado e Economia. http://colunistas.ig.com.br/guilhermebarros

[2] Verdier, S., Coutinho, J.A.P., Silva, A.M.S., Alkilde, O.F. and Hansen, J.A. (2009) Fuel, 88, 2199-2206. http://dx.doi.org/10.1016/i.fuel.2009.05.016

[3] Teixeira, S.C.S. (1994) Estudo Comparativo de Polímeros como Melhoradores de Índice de Viscosidade. M.Sc. Dissertation, Federal University of Rio de Janeiro, Rio de Janeiro.

[4] Kraguljać, K., Šoljić, I., Vidović, E. and Jukić A. (2009) Chemical Engineering Transactions, 17, 1741-1746.

[5] Lynch, T.R. (2008) Process Chemistry of Lubricant Base Stocks. CRC Press, Boca Raton.

[6] Mortier, R.M., Fox, M.F. and Orszulik, S.T. (2010) Chemistry and Thecnology of Lubricants. 3rd Edition, Springer, London. http://dx.doi.org/10.1023/b105569

[7] Jukić, A., Rogošić, M., Frankjić, I. and Šoljić, I. (Eds.) (2009) European Polymer Journal, 45, 2594-2599. http://dx.doi.org/10.1016/j.eurpolymj.2009.06.014

[8] Wanderley, A.M.R.F. (2002) Caracterização Toxicológica dos Óleos Parafínicos Produzidos pela Petrobras (Toxicologic Characterization of Paraffinic Oils Produced by Petrobras). External Report, CENPES/Lubrificantes e Produtos Especiais, Rio de Janeiro.

[9] Coutinho, F.M.B. and Teixeira, S.C.S. (1993) Polymer Testing, 12, 415-422. http://dx.doi.org/10.1016/0142-9418(93)90013-F

[10] Silva, A.E.O. (2011) Transposição Didática: A Química dos Óleos Lubrificantes. B.Sc. Monography, Brasília University, Brasília.

[11] Ver Strate, G. and Struglinski, M.J. (1991) Polymers as Lubricating-Oil Viscosity Modifiers. In: Polymers as Rheology Modifiers. Proceedings of the ACS Symposium Series, 462, American Chemical Society, 256-272. http://dx.doi.org/10.1021/bk-1991-0462.ch015

[12] Jukić, A., Tomašek, L.J. and Janović, Z. (2005) Lubrication Science, 17, 431-449. http://dx.doi.org/10.1002/ls.3010170406

[13] Jukić, A., Vidović, E. and Janović, Z. (2007) Chemistry and Technology of Fuels and Oils, 43, 386-394. http://dx.doi.org/10.1007/s10553-007-0068-9

[14] Schramm, G. (2006) Reologia e Reometria. Artliber Editora Ltda., São Paulo. 
Scientific Research Publishing (SCIRP) is one of the largest Open Access journal publishers. It is currently publishing more than 200 open access, online, peer-reviewed journals covering a wide range of academic disciplines. SCIRP serves the worldwide academic communities and contributes to the progress and application of science with its publication.

Other selected journals from SCIRP are listed as below. Submit your manuscript to us via either submit@scirp.org or Online Submission Portal.
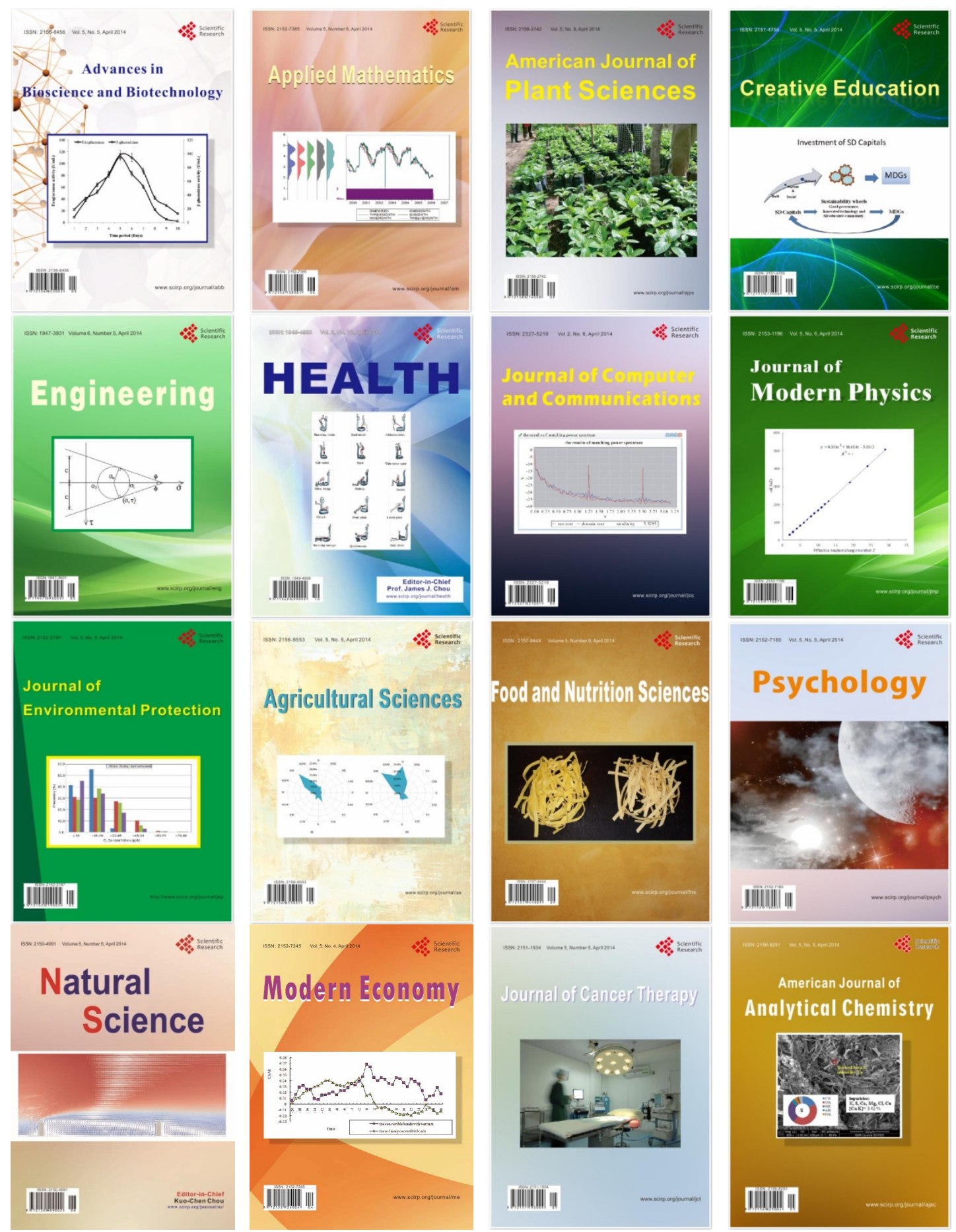